Results This study has provided preliminary data on the perception of Saudi mothers who have a child with CP. It was found that mothers reflected a good understanding about the medical terminology of the CP. However, most of the mothers did not accept that their child had this diagnosis. There was also a strong belief that the children were experiencing developmental delay. Mothers expressed their trust in God, and relying on God for their child cure and health and for giving them the courage to care for the child.

Conclusions This work has allowed mothers to express their beliefs about the term CP. The data yielded information regarding mothers' beliefs surrounding the meaning of the CP term. These ranged from traditional and cultural beliefs to medical explanations, and to frank confusion between the two.

\section{ASSESSMENT OF DATA RECORDING OF KEY FACTORS RELATING TO DIAGNOSIS, INVESTIGATION, TREATMENT AND MULTI-PROFESSIONAL INPUT FOR PATIENTS WITH CEREBRAL PALSY}

doi:10.1136/archdischild-2012-302724.1754

G Nepali, E Bolland. Community Paedaitrics, Chase Farm Hospital, Enfield, UK

Aims To assess the quality and availability of data recorded within clinic letters regarding diagnosis, classification, investigation and management of Cerebral Palsy (CP).

Methods A retrospective review of case notes on the most recently reviewed $(n=50)$ patients within community paediatric catchment area with a diagnosis of CP, assessing quality of data recorded in the last clinic letter.

Results Mean age at first referral was 2.06 years. $64 \%$ had Spastic CP, with $74 \%$ having bilateral symptoms. GMFCS score was recorded in $54 \%$ and MACS score in $0 \%$. Specific areas of management varied dramatically: input recorded physiotherapy $70 \%$, psychological $6 \%$.

$30 \%$ of letters noted cognitive skills, $34 \%$ visual assessment, $12 \%$ hearing status, $36 \%$ presence of epilepsy, $32 \%$ MRI findings, 14\% hip-x-ray, 22\% SALT involvement and 32\% OT involvement.

Conclusion The data suggests that there is currently poor written communication of functionality and involvement of multiple professional groups in this sample of children with CP, although diagnostic elements are better recorded.

The information required may be elsewhere not readily apparent within the notes. Therefore it would not be safe to assume that absence of details signifies lack of professional involvement or normal functionality.

This information is critical to understanding patient needs, especially since patients may be seen by different healthcare professionals with limited handover. Lack of such clear communication makes it difficult to audit sub-groups of patients and identify the quality of care being provided.

Subsequent to this audit outcome documentation template for clinic reviews for children with CP has been developed.

\section{PARENTS' EXPERIENCES WITH A PREVENTIVE INTERVENTION FOR VERY LOW BIRTH WEIGHT INFANTS AND THEIR PARENTS AFTER DISCHARGE FROM HOSPITAL}

doi:10.1136/archdischild-2012-302724.1755

$\mathrm{M}$ Jeukens-Visser, $\mathrm{P}$ van Schie, K Koldewijn, $\mathrm{G}$ van der Straeten, MJ Wolf. AMC, Amsterdam, The Netherlands

Background and Aims The ToP programme is a preventive, neurobehavioral intervention for very low birth weight infants and their parents. It is provided at home by a specially trained paediatric physical therapist from discharge until 1 year. The aim was to investigate parents' experiences with the ToP programme.
Methods All parents participating in the ToP programme were asked to fill in two questionnaires at the end of the intervention: the Measure of Processes of Care (MPOC-20), a measure of parental perception of the extent to which the services they received were family-centered and a questionnaire on the satisfaction of the parents with the ToP programme.

Results From the 124 parents who completed the intervention, 74 returned the questionnaires $(60 \%)$. Children had a mean (SD) birthweight of 1287 (377) grams and a mean (SD) gestational age of 29.8 (2.6) weeks. Mean age (SD) of mothers at birth was 31.5 (5.2) years. The mean (SD) domain scores (scale 1-7) of the MPOC were high and ranged from 5.5 (1.4) for providing specific information to 6.3 (0.5) for coordinated and comprehensive care. Even though $27 \%$ reported not to have known what to expect from the intervention, the parents rated the ToP programme a mean (SD) of 9.0 (1.0) on a scale from 0-10. Parents were positive about the knowledge of the therapist, the suggestions they received, and the number of visits (mean (SD) 9.3 (2.0)).

Conclusions Parents were very positive about the ToP programme. It was perceived as respectful, supportive, and well coordinated.

\section{CAN CHILDREN WITH DISABILITIES BE TRANSPORTED SAFELY? THE CHALLENGE AND SOLUTIONS}

doi:10.1136/archdischild-2012-302724.1756

'M Bull, ' J O'Neil, ' J Talty, J Yonkman, MS, ${ }^{2}$ OTR. 'Department of Pediatrics, Section of Developmental Pediatrics, IU School of Medicine, Riley Hospital for Children at IU Health; '2Automotive Safety Program, Riley Hospital for Children at IU Health, Indianapolis, IN, USA

Background and Aims Children with diverse medical problems are transported daily to school, medical appointments and family events. One-in-ten children worldwide have conditions that may require special consideration to ensure optimal transportation safety. How to safely transport this population is poorly understood. This initiative enhances understanding of correct occupant protection for children with disabilities.

Method Positioning problems and physiologic stability of children with developmental, skeletal, and physiologic abnormalities and behavioral concerns were reviewed and where needed special restraints were identified. Biomechanical principles were investigated and applied where possible. Solutions were sought for each condition involving a unique concern. A literature review was conducted and resources identified, many of which were developed by the authors.

Results Many infants and children with low birth weight or born prematurely, cerebral palsy, hypotonia, or spina bifida, and skeletal problems including children in casts following injury or surgery frequently cannot be safely transported in conventional car seats. Even within developed nations children with disabilities are suboptimally restrained. Solutions for difficult transportation problems have been developed and identified. Some problems remain unresolved and safety remains suboptimal for some conditions.

Conclusions Motor vehicle crashes are a leading cause of mortality and morbidity worldwide and children are often severely injured or killed. Appropriate use of child restraints has been shown to save lives and reduce injury. Solutions exist for many conditions and families benefit from assistance in identification and access to these solutions. Transportation options will be explained and care improved by the information in this presentation.

\section{MECHANICAL VIBRATIONS DURING SIMULATED NEONATAL TRANSPORT}

doi:10.1136/archdischild-2012-302724.1757

${ }^{1} \mathrm{~B}$ Schwaberger, ${ }^{1} \mathrm{G}$ Pichler, ${ }^{1} \mathrm{~B}$ Urlesberger, ${ }^{2} \mathrm{~A}$ Hohl, ${ }^{2} \mathrm{H}$ Pessenhofer, ${ }^{2} \mathrm{~B}$ Kohla, ${ }^{3} \mathrm{G}$ Köstl, ${ }^{3} \mathrm{R}$ Kerbl. 'Department of Pediatrics; 'Institute of Physiology, Medical University of Graz, Graz; ${ }^{3}$ Department of Pediatrics, LKH Leoben, Leoben, Austria 